\title{
Laser Effects in Semiconductor Heterostructures Within an Extended Dressed-Atom Approach
}

\author{
H.S. Brandi ${ }^{a}$, A. Latgé $^{b}$, and L.E. Oliveira ${ }^{c}$ \\ ${ }^{a}$ Instituto de Física, Univ. Federal do Rio de Janeiro, Rio de Janeiro - RJ, 21945-970, Brazil \\ ${ }^{b}$ Instituto de Física, Univ. Federal Fluminense, Niterói - RJ, 24210-340, Brazil \\ ${ }^{c}$ Instituto de Física, Unicamp, CP 6165, Campinas - São Paulo, 13083-970, Brazil
}

Received on April 22, 2001

\begin{abstract}
We extend the dressed-atom approach to treat the interaction of a laser field with a semiconductor system. The semiconductor is modeled via a simple Kane band-structure scheme and the interaction with the laser field is incorporated through the renormalization of the semiconductor energy gap and conduction/valence effective masses. Far from resonances, such one-body approach allows the study of the effects of laser fields on a variety of optoelectronic phenomena in semiconductor systems for which the effective-mass approximation provides a good physical description. We calculate the effects originated by the laser-dressing on the donor and exciton peak energies in quantum-well heterostructures, and show that they may be quite considerable and observable.
\end{abstract}

\section{Introduction}

The possibilities to design new efficient optoelectronic switches and modulators may be greatly improved if we are able to understand the basic physics involved in the external field-semiconductor interaction. A considerable amount of work has been devoted to the study of the interaction of external electric and magnetic fields [1] with semiconductor quantum wells and superlattices, and the physical processes are now well understood. A more complicated situation occurs when light interacts with a semiconductor, and a detailed description of the laser-semiconductor interaction requires a many-body treatment, as excitations (real or virtual) such as excitons are created and interact via Coulomb forces $[2,3]$.

In the present work we study the effects of a laser beam in the exciton states in semiconductor heterostructures. We also discuss how an homogeneous and monochromatic laser beam modifies the impurity states of $\mathrm{GaAs}-(\mathrm{Ga}, \mathrm{Al}) \mathrm{As}$ semiconductor quantum wells (QWs) under applied magnetic fields perpendicular to the QW interfaces. A realistic description of the laser effects on shallow-impurity states in semiconductor heterostructures under magnetic fields would, in principle, require complex calculations involving, for instance, Coulomb interactions between impurities and all virtual excitonic states. Nevertheless, a much simpler situation occurs when the laser is tuned far from any resonances, since the main physics of the laser-semiconductor interaction may be theoret- ically described by using a non-perturbative one-body approximation $[4,5,6]$. From the experimental point of view, the shifts on the impurity levels induced by the laser light may be used as a possible application to ultrafast opto-electronic devices where no photon absorption occurs in the device.

Here we consider a description of the semiconductor system which includes spin-orbit coupling within the Kane model [7]. The calculations are based on a steady-state model whereas actual experiments dealing with changes in the electronic structure of the semiconductor system are performed using ultrafast laser pulses and intensities of $\mathrm{GW} / \mathrm{cm}^{2}$ (the pulsed-laser regime in which the agreement with steady-state results is expected has been extensively investigated both theoretically and experimentally [3]).

\section{Laser-dressed 3-band Kane model}

We adopt the Kane model to describe the set of states formed by the lowest conduction band $\left(\Gamma_{6}\right)$, the highest light- and heavy-hole valence bands $\left(\Gamma_{8}^{l h, h h}\right)$, and the splitted spin-orbit band $\left(\Gamma_{7}\right)$, which is splitted from the other two degenerated valence bands by $\Delta$. In what follows, we use as a basis the states obtained from the diagonalization of the Kane matrix [8]. The effect of a homogeneous laser field on the band structure may be obtained from the Hamiltonian $[4,5]$ 


$$
H=H_{o}+\hbar \omega a^{+} a+\frac{e}{m_{o} c} A_{\omega} \hat{p} \cdot \hat{\epsilon}\left(a^{+}+a\right),
$$

where $H_{o}$ is the diagonal matrix obtained from the Kane model, and $a^{+}(a)$ is the creation (annihilation) photon operator associated with the laser mode of frequency $\omega$ and polarization $\hat{\epsilon}$. The vacuum field amplitude in the volume $\Omega$ is given by $A_{\omega}=\left(\frac{2 \pi \hbar c^{2}}{\omega \Omega}\right)^{\frac{1}{2}}$ which is related to the classical amplitude of the photon vector potential $A_{o}$ by $A_{o}=2\left(N_{o}\right)^{1 / 2} A_{\omega}$ for $N_{o}>>1$, where $N_{o}>>1$ is the average number of photons in the field. Using the states obtained from the diagonalization of the Kane matrix, it is straightforward to extend the dressed-atom approach [9] and diagonalize the Hamiltonian of eq. (1) within the $\left|\Gamma_{8}, N+1\right\rangle \equiv$ $\left|\Gamma_{8}>\otimes\right| N+1>$ and $\left|\Gamma_{6}, N>\equiv\right| \Gamma_{6}>\otimes \mid N>$ manifold, where $\mid N>$ represents a Fock state with $N \approx N_{o}$ photons [9, 10].

From the diagonalization of the Hamiltonian, one obtains [6] the associated laser-dressed conduction (+) and valence (-) electronic bands, i.e.,

$$
\begin{aligned}
\epsilon_{ \pm}=\frac{\epsilon_{o} \pm \hbar \omega}{2}+\frac{\Lambda_{o}^{2}}{6 \delta^{\prime}} & \pm \frac{1}{2} \sqrt{\frac{8 \Lambda_{o}^{2}}{3}+\left(\delta+\frac{\Lambda_{0}^{2}}{3 \delta^{\prime}}+\frac{4 \Lambda_{0}^{2}}{3 \Lambda_{1}}\right)^{2}} \\
& +\frac{\hbar^{2} k^{2}}{2 m_{ \pm}}
\end{aligned}
$$

and corresponding renormalized effective masses $m_{ \pm}$ (see Brandi et al [6]). In the above expression, $\epsilon_{o}$ is the semiconductor energy gap [8], $\delta$ is the laser detuning given by $\epsilon_{o}-\hbar \omega, \delta^{\prime}=\delta+\Delta$, and $\Lambda_{1}=\epsilon_{o}+\hbar \omega$. The characteristic energy associated to the laser-semiconductor interaction is related to $\Lambda_{o}=\left(\frac{e A_{o}|p|}{2 m_{\circ} c}\right)$.

Note that the k-dependent semiconductor energy gap is dressed by laser effects, and is given by the difference between the above renormalized conduction and valence electronic bands

$\tilde{\epsilon_{o}}(k)=\epsilon_{o}-\delta+\sqrt{\frac{8 \Lambda_{o}^{2}}{3}+\left(\delta+\frac{\Lambda_{0}^{2}}{3 \delta^{\prime}}+\frac{4 \Lambda_{0}^{2}}{3 \Lambda_{1}}\right)^{2}}+\frac{\hbar^{2} k^{2}}{2 \mu}$,

with $\frac{1}{\mu}=\frac{1}{m_{+}}-\frac{1}{m_{-}}$.

The above equations provide the framework for calculating laser effects on semiconductor systems within the Kane band-structure picture.

The dependence of the laser-dressed conductionand valence-band effective masses and the corresponding behavior of the dressed $k=0$ semiconductor energy gap [cf. eq.(3)] are shown in Figure 1 (where $[4,5]$ $I_{o} \approx 5 \times 10^{7} \mathrm{MW} / \mathrm{cm}^{2}$ ) for a laser detuning $\delta=0.05$ $\epsilon_{o}$, and parameters corresponding to bulk GaAs. It is clear that laser effects correspond to a renormalization both of the semiconductor energy gap and of
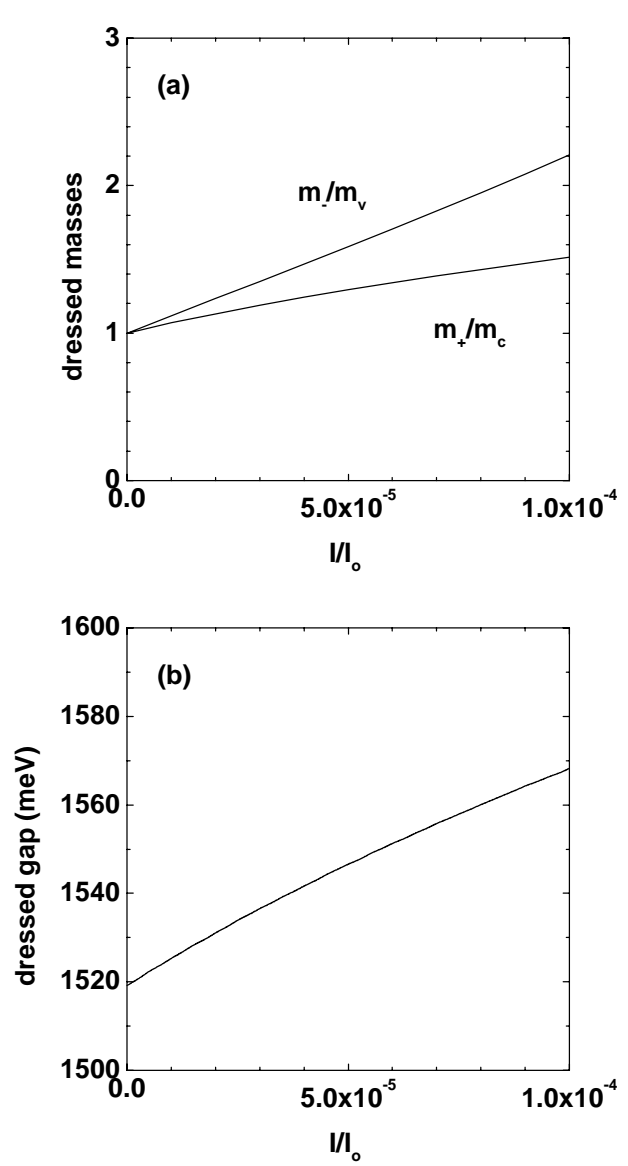

Figure 1. GaAs laser-dressed effective masses for the conduction $\left(m_{+}\right)$and valence $\left(m_{-}\right)$bands, and energy gap. Results display the dependence on the laser intensity for a fixed detuning $\delta / \epsilon_{0}=0.05$.

the conduction/valence effective masses. Also, a comparison of the present results with the previous calculation based on a two-band model semiconductor $[4,5]$ indicates that results are qualitatively the same, the differences increasing for smaller detunings and high laser intensities, as one would expect. Therefore, we may infer that laser dressing does not strongly depend on the modelling of the band structure, and therefore the present Kane-model renormalized effective-mass approach, valid within the one-particle picture and for a laser tuned far from any resonances, may be used to provide an adequate indication of the laser effects on any semiconductor heterostructure for which the effective-mass approximation is a good physical description.

\section{Laser-induced exciton and donor shifts in GaAs- (Ga,Al)As quantum wells}

As a simple application of the renormalization scheme, we calculate the optical Stark shift of exciton states 
in a $100 \AA G a A s-G a_{0.7} A l_{0.3} A s$ QW. The 1s-like exciton ground state was evaluated within a fractionaldimensional scheme [11] using the 3-band Kane renormalized GaAs conduction/valence effective mass and dressed band gap. Results for the laser-intensity dependence of the exciton shift (and peak energy) are presented in Fig. 2 (and Fig. 3) for a laser tuned far from exciton resonances, i.e., $\delta=0.05 \epsilon_{o}$. The blue laser-induced shift in the exciton peak energies is quite remarkable, and calculated results qualitatively agree with femtosecond measurements by Mysyrowicz et al [12]. The Kane-model contributions to the exciton Stark shift are due to the laser-induced changes in the GaAs gap, exciton binding energy, and QW confinement both of free electrons and holes. It is apparent from Fig. 2 that the main contribution to the exciton blue shift is essentially due to laser-induced changes in the GaAs semiconductor gap. Notice that, in the large detuning limit, perturbation theory (PT) in the laserdressed 3-band Kane gap results in an exciton blue shift given by $\frac{4 \Lambda_{o}^{2}}{3 \delta}$, in contrast with the 2-band model approach $[4,5]$ which gives a larger detuning $\mathrm{PT}$ result of $\frac{2 \Lambda_{o}^{2}}{\delta}$. This 2-band PT result for the contribution of the dressed gap to the exciton shift corresponds to the same exciton blue shift, in the large detuning limit, obtained through a many-body diagrammatic derivation and interpreted by Combescot [2] as coming from Pauli exclusion between the two e-h pairs forming the biexciton. We mention therefore that the present Kane results demonstrate that dressed-band effects within a 3band model calculation would change the zeroth-order term of the 2-band many-body diagrammatic approach [2], and suggest that the contribution due to more realistic band-dressing calculations may be as important as many-body corrections. Also, the present renormalized approach for evaluating the exciton binding energy corresponds essentially to a diagrammatic summation of ladder diagrams associated with laser-dressed e-h bubbles built from a renormalized electron and a renormalized hole in the presence of the Coulomb interaction.

As a second application, we study the laser effects on the on-center donor-impurity states of a $100 \AA$ $G a A s-G a_{0.7} A l_{0.3} A s$ QW under the presence of an applied magnetic field perpendicular to the QW interfaces [1]. We follow a standard variational scheme within a renormalized laser-dressed effective-mass approach and choose a 1s-like on-center trial envelope wave function as a product of the exact solution of the square well QW potential and a hydrogenic-like variational 1s function [1]. We consider the effects of a laser on the 1s-like shallow donor state under the presence of an applied magnetic field perpendicular to the QW interfaces. Results for the magnetic-field dependence of the donor peak energy (transitions from the 1s-like donor to the first valence mini-band) are displayed in Fig. 4 for $I / I_{o}=10^{-4}$ and a detuning parameter $\delta=0.05 \epsilon_{o}$. One notices that the laser-dressed shift in the donor peak energy is of the same order of magnitude of the QW confined donor binding energy, and much stronger than the corresponding shift induced by the applied magnetic field. The strong blue shift on the donor peak energy could be easily observable, although, to our knowledge, there are no experimental measurements concerning laser effects on impurity states in doped GaAs-GaAlAs QWs.

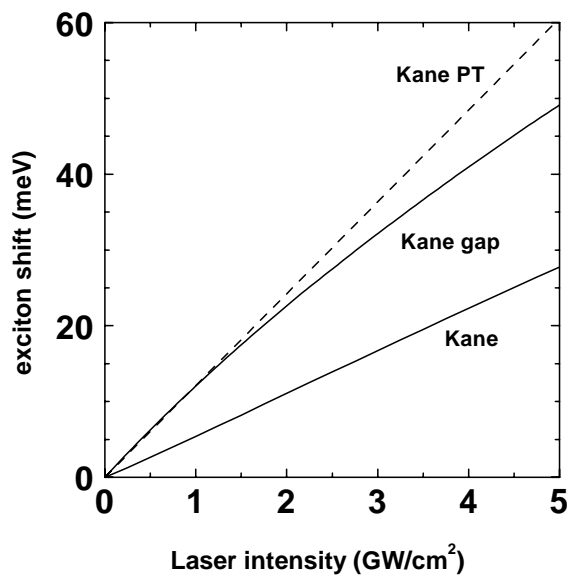

Figure 2. Laser-intensity dependence of the exciton optical Stark shift for a $100 \AA G a A s-G a_{0.7} A l_{0.3} A s$ QW and a laser detuning $\delta / \epsilon_{0}=0.05$. The full curves represent the Kane-model contributions to the exciton Stark shift due to the laser-induced changes in the GaAs gap (labelled "Kane gap"), and the total optical exciton Stark shift shown as a full curve (labelled "Kane"). It is also shown the perturbative (Kane PT) results (dashed curve).

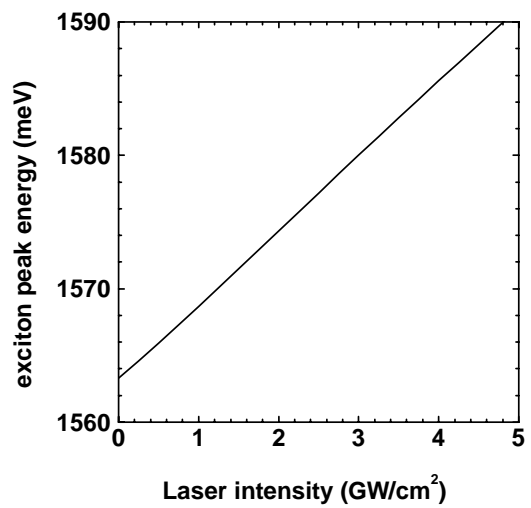

Figure 3. Laser-dressed exciton-peak energy for a $100 \AA$ $G a A s-G a_{0.7} A l_{0.3} A s$ QW as a function of the laser intensity and for a detuning parameter $\delta=0.05 \epsilon_{o}$.

\section{Conclusions}

We have presented a theoretical approach of the laserfield effects on semiconductor low-dimensional systems by adopting a picture in which the light-matter interaction is taken into account by dressing the semiconductor energy gap and conduction/valence effective masses. Both the two-band and three-band Kane semiconductor models for the electronic band structure are 
shown to give essentially the same qualitative results for the laser dependence of the dressed semiconductor gap and corresponding effective masses, although an appropriate quantitative comparison with experiment would certainly require a more realistic description of the semiconductor band structure.

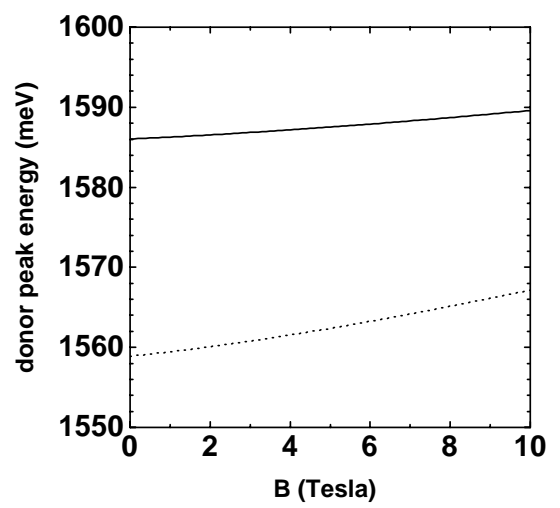

Figure 4. Laser-dressed on-center donor-peak energies for a $100 \AA \mathrm{GaAs}-\mathrm{Ga}_{0.7} \mathrm{Al}_{0.3} A s$ QW for $I / I_{o}=10^{-4}$ and $\delta=0.05 \epsilon_{o}$. Dotted lines are the corresponding undressed results.

\section{Acknowledgments}

The authors would like to thank the Brazilian Agencies CNPq, FUJB-UFRJ, FAPERJ, FAPESP, and FAEP-UNICAMP for partial financial support.

\section{References}

[1] A. Latgé, N. Porras-Montenegro, M. de Dios-Leyva, and L. E. Oliveira, Phys. Rev. B53, 10160 (1996); F.
J. Ribeiro, A. Latgé, and L. E. Oliveira, J. Appl. Phys. 80, 2536 (1996).

[2] M. Combescot, Phys. Reports 221, 167 (1992).

[3] M. Lindberg and S. W. Koch, Phys. Rev B 38, 7609 (1988); C. Ell, J. F. Miller, K. El Sayed, and H. Haug, Phys. Rev. Lett. 62, 304 (1989); S. M. Sadeghi, J. F. Young, and J. Meyer, Phys. Rev. B 56, R15557 (1997).

[4] H. S. Brandi, A. Latgé, and L. E. Oliveira, Sol. State. Commun. 107, 31 (1998); ibid., Phys. Stat. Sol. (b) 210, 671 (1998).

[5] H. S. Brandi and G. Jalbert, Sol. State Commun. 113, 207 (2000); H. S. Brandi, A. Latgé, and L. E. Oliveira, Physica B: Phys. of Cond. Matter (in press).

[6] H. S. Brandi, A. Latgé, and L. E. Oliveira, Sol. State Commun. 117, 83 (2001); ibid., Phys. Rev. B (in press, July 15, 2001).

[7] E. O. Kane in "Narrow Gap Semiconductors. Physics and Applications", ed. by W. Zawadzki, Lec. Notes Phys. 133 (Springer Verlag, Berlin, 1980).

[8] G. Bastard, "Wave mechanics applied to semiconductor heterostructures". Les Editions de Physique, Les Ulis, 1988.

[9] C. Cohen-Tannoudji, J. Dupont-Roc, and G. Grynberg, "Processus d'interaction entre photons et atomes", Editions du CNRS, 1988.

[10] G. Jalbert, B. Koiller, H. S. Brandi, and N. Zagury, J. Phys. C 19, 5745 (1986).

[11] A. Matos-Abiague, L. E. Oliveira, and M. de DiosLeyva, Phys. Rev. B 58, 4072 (1998); E. Reyes-Gómez, A. Matos-Abiague, C. A. Perdomo-Leiva, M. de DiosLeyva, and L. E. Oliveira, ibid. 61, 13104 (2000).

[12] A. Mysyrowicz, D. Hulin, A. Antonetti, A. Migus, W. T. Masselink, and H. Morkoç, Phys. Rev. Lett. 56, 2748 (1986). 